\title{
Retraction Note to: A fuzzy multi-objective immune genetic algorithm for the strategic location planning problem
}

\author{
Xiao Zhao ${ }^{1,3} \cdot$ Xuhui Xia $^{1} \cdot$ Lei Wang ${ }^{1,2} \cdot$ Jianhua Cao ${ }^{1,2}$
}

Published online: 18 January 2022

(C) Springer Science+Business Media, LLC, part of Springer Nature 2022

\section{Retraction to: Cluster Computing (2019) 22:S3621-S3641 https://doi.org/10.1007/ s10586-018-2212-1}

The Editor-in-Chief is retracting this article at the request of the corresponding author Lei Wang because the model, the algorithm and the results presented are the work of Liu Aijun and Hu Hesuan of Xidian University. The Associate
Dean (Research), School of Economics and Management, Xidian University, has confirmed that the intellectual property in this work belongs to Xidian University. Xiao Zhao, Xuhui Xia and Jianhua Cao have not responded to correspondence from the Publisher about this retraction.

Publisher's Note Springer Nature remains neutral with regard to jurisdictional claims in published maps and institutional affiliations.

The original article can be found online at https:// doi.org/10.1007/s10586-018-2212-1.

Lei Wang

wengufei0565@163.com

1 Key Laboratory of Metallurgical Equipment and Control Technology (Wuhan University of Science and Technology), Ministry of Education, Wuhan 430000, China

2 Center of Service Science and Engineering, Wuhan University of Science and Technology, Wuhan 430000, China

3 School of Mechanical Engineering, Hubei University of Arts and Science, Xiangyang 430062, Hubei, China 